\title{
LES PLUIES OCÉANIQUES CLASSIQUES DANS L'EST DE LA FRANCE ET L'AVERSE DES 28 ET 29 DÉCEMBRE 1947 EN LORRAINE ET EN ALSACE
}

\author{
(COMMUNICATION PRESENTÉE AU COMITÉ TECHNIQUE DE LA SOCIÉTÉ HYDROTECHNIQUE \\ DE FRANCE, LE 4 JUIN 1948)
}

\section{par Maurice PARDE}

Professeur d̀ l'Ecole des Ingénieurs Mydrauliciens et à l'linstitut de Géographie Alpine de Grenoble, Vice-Président de la Commission $d^{\prime}$ "Hydrologie » du Comité Technique de la S. H. F.

Le 29 et le 30 décembre 1947 ont apporté d̀ la Lorraine, et dans une moindre mesure à I'Alsace, une catastrophe extraordinaire par "le fait d'inondations supérieure à toutes celles dont on a souvenir sur la Moselle jusque vers Thionville, sur la Meurthe depuis les abords de Lunéville, sur la Meuse dans la région de Verdun, et aussi sur la Sarre supérieure.

En Alsace aussi, les rivières ont commis beaucoup de ravages, mais le phénomène parait avoir été moins violent.

Je m'étais offert aux Pouvoirs Publics pour étudier en détail les causes, le développement, les caractéristiques de ce désastre, comme je l'avais fait pour les inondations mémorables de Mars 1930 dans le Languedoc et l'Aquitaine et d'Octobre 1940 dans les Pyrénées Orientales. Je me proposais, pour suppléer au nombre forcément bien trop faible des échelles, d'adresser une circulaire-questionnaire aux Officiers et Agents forestiers des zones atteintes par la crue et aux instituteurs des localités riveraines. Cette méthode m'avait, lors des deux occasions pré. cédentes et surtout pour les phénomènes d'Octobre 1940, procuré des renseignements de toute première valeur, car un certain nombre de personnes consultées avaient révélé des qualités éminentes d'observation et de réflexion.

Mais on a préféré faire appel pour cette recherche aux compétences locales. Je n'ai plus qu'ò attendre le résultat de leurs investigations pour acquérir une opinion approfondie sur le phénomène. Mais dès mainienant je puis four.. nir sur ses causes atmosphériques des renseigne. ments assez précis. lis me proviennent surtout de deux sources: d'une part, M. SAMSON, le très distingué et toujours obligeant Sous-Directeur de la Météorologie Nationale a bien voulu me faire copier et $m$ 'adresser des chiffres et des remarques nombreuses sur les observations de toutes sortes. Et d'autre part, vers la fin de Janvier, M. ROTHÉ, Professeur à la Faculté des Sciences de Strasbourg et Directeur en cette ville de f'Institut de Physique du Globe, ignorant que j'avais abardonné mon enquête, m'a adressé une note bien documentée et des plus sagaces sur les averses et les fontes de neige qui ont causé la crue. Ce distingué Collègue souhaitait que je publiasse son texte en introduction de mon rapport, ce que j'aurais fait avec le plus grand plaisir, en ajoutant quelques commentaires, notamment des remarques comparatives. Comme j'avais renoncé à composer un mémoire, j'ai conseillé à mon collègue de publier séparément son étude. Elle a paru dans les «Annales de Géographie » (i), mais je tiens à spécifier que je lui emprunte beaucoup des données que je vais exposer cidessous.

\section{1. - TYPE DE LA CRUE}

Indiquons d'abord le fait essentiel que cette crue appartient de façon indiscutable au type bien connu de ce que j'appelle les crues océaniques classiques. Ces phénomènes, surtout fré-

(1) ROTHÉ J.-P. - Le's causes météorologiques des inondations de décembre 1947 dans le nord-est de la France. Annales de Géographie, 57" année, iuillet-sept. 1948, pages $205-211,2$ figures. 
quents en saison froide, ont pour cause, avec ou sans renfort de fontes nivales, des pluies à vaste extension, et qui durent dans les cas simples de 1 à 3 jours sur de très vastes étendues, puisqu'el. les peuvent faire monter à la fois le Lot, le Tarn inférieur, la Dordogne, la Loire inférieure, la Seine, la Meuse, la Moselle, le Neckar, le Main, le Rhin, la Saône et le Doubs, le Rhône supérieur et l'Ain, I'Isère, le Drac inférieur, etc., et même dans certains cas tout à fait grandioses (Mars 1896, Décembre 1882, 1918), le Danube superieur avant Vienne, la Vltava (Moldau) et l'Elbe.

La pluie, qui cause ces débordements, n'est que très rarement corsée par des orages. Elle est du type continu, quoique variable en intensité d'une heure à l'autre Imais là-dessus nous manquons des précisions que fournissent en d'autres pays, aux Etats-Unis par exemple ou en Italie, de nombreux pluviomètres enregistreurs). Les pluies s'accompagnent en hiver de températures très tièdes (maxima de 10-à $15^{\circ}$ dans les régions basses). Dans les cas plus rares où elles survien. nent en été, les températures ambiantes sont au contraire fraîches, ou plutôt elles donnent cette impression sans présenter de chiffres inférieurs à ceux de saison froide (c'est en général le contraire) parce qu'elles surviennent généralement après des journées plus chaudes.

Les vents qui charrient les nimbus dont ces averses tombent ( ciel couvert avec pluie » selon les termes de la Météorologie Nationale: sont parfois très violents. Mais ce n'est pas une règle. Ils ont immanquablement une composante ouest. Plus exactement ils viennent de sud-ouest ou d'ouest-sud-ouest. Quand ils émanent de directions autres, soit de sud-sud-ouest, ou sud, soit de nord-ouest (2), on constate assez fréquemment une distribution anormale de l'averse.

J'appelle normale une répartition réglée surtout par les inégalités du relief, ovec grosses chutes d'eau sur les versants occidentaux des lignes orographiques plus ou moins perpendiculaires au vent, et précipitations plus maigres ou infimes dans les dépressions. (Ainsi en janvier 1910 dans le bassin du Rhône, en 3 jours c̀ peu près $270 \mathrm{~m} / \mathrm{m}$ à Thônes (Hte-Savoie), 28 1 à Morez (Jura), 289 à Mouthe (Doubs), contre 2 à Lyon ; en 4 jours de janvier.1899, 213 à Thônes, 180 à St-Claude, 222 à Allemont (Isère), 5 à Lyon.

(2) Le vent de Nord-Ouest marque plutôt la fin de I'averse continue, génératrice de ruissellement grave, et le début de giboulées, d'averses éparșes avec refroidissement. Cependant les pluies pyrénéennes classiques, qui influencent la Garonne supérieure, I'Adour, I'Agout, et le bas Tarn (catastrophe de Juin 1875), s'accompagnent tout en restant continues, de vents à composante nord-occiden tale. Les pluies méditerranéennes en France ont pour cause des vents de sud à est, et presque toujours de Sud-Est.

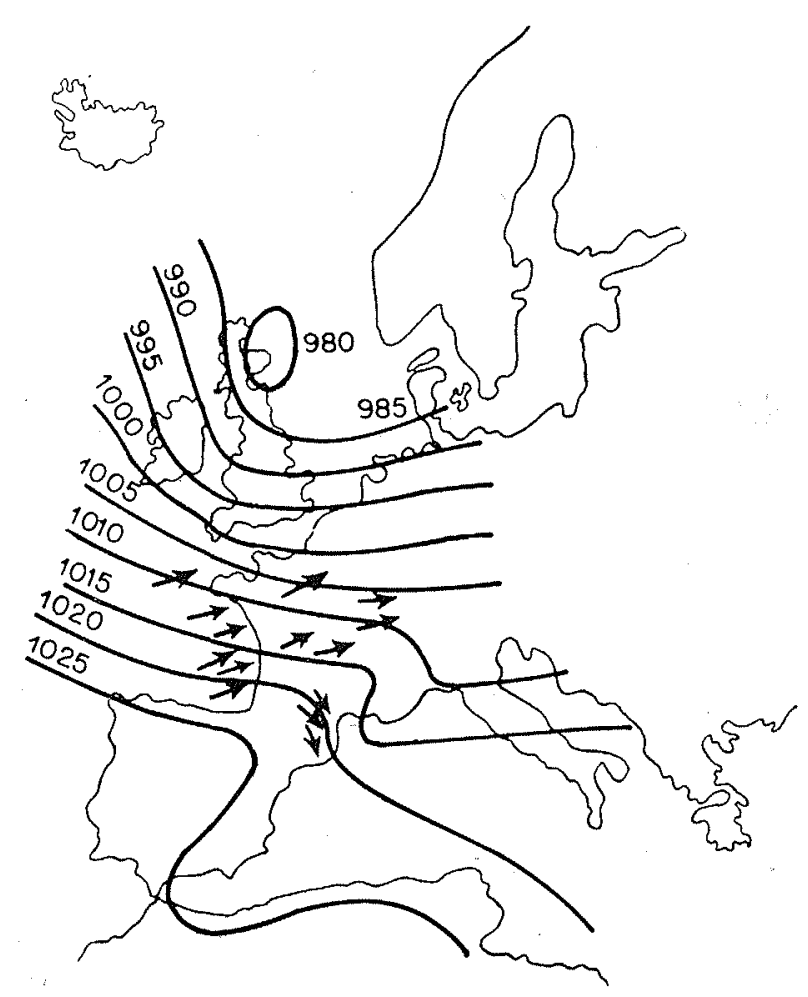

Fig. 1. - Situation barométrique du 23 décembre 1918 à 7 heures (pressions en millibars).

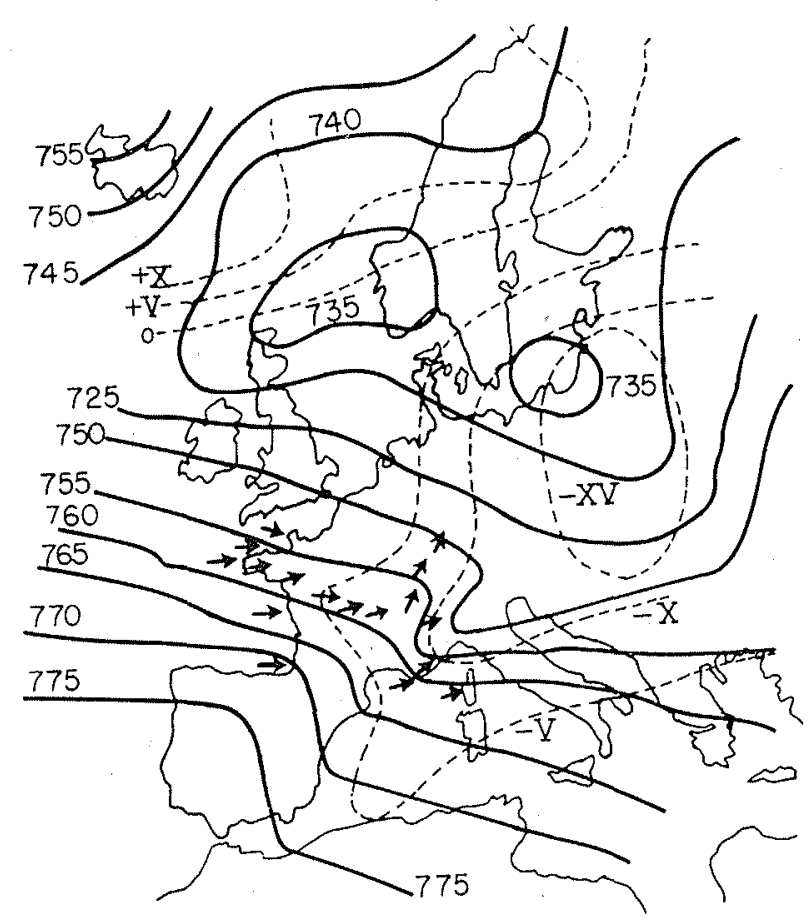

Fig. 2. - Situation barométrique du 19 janvier 1910 à 7 heures (pressions en $\mathrm{mm}$.)

Situations atmosphériques dangereuses et typiques pour les crues océaniques. 
Au cas de distribution anormale, au sens que j'entends et surtout quand le vent se rapproche de la direction sud-nord, on observe généralement des pluies moins fortes sur les reliefs, moins faibles ou même copieuses dans les plaines ou les creux. Il arrive même que l'averse ait le maximum de violence dans les stations abritées, ma! arrosées lors des cas normaux. Je soupçonne qu'alors agissent surtout, avec ou sans orages, des phénomènes frontaux, c'est-à-dire liés à l'existence de fronts ou plans obliques de contact entre masses d'air thermiquement hétérogènes par exemple, air tiède de sud-ouest, vent frais de nord-ouest. Ainsi lors de l'averse d'octobre 1888, très dommageable pour le haut Rhône avant I'Ain et pour I'Arve, on nota en un jour, $160 \mathrm{~m} / \mathrm{m}$ à Evian, 137 à Bonneville, 135 à Cruseilles (Hte-Savoie), stations généralement peu arrosées; et en trois jours $42 \mathrm{~m} / \mathrm{m}$ à Sallanches, 82 à Taninges, postes ordinairement bien plus éprouvés. En novembre 1913. (grande crue de la Saône), la région basse vers Chalon, Auxonne, Besançon, fut plus arrosée par le Jura.

Les crues océaniques classiques correspondent aux conditions isobariques suivantes:

une dépression fermée passe sur la mer du Nord, un anticyclone se bombe vers le nord-est, sur la péninsule ibérique, avec une direction sud-ouestnord-est pour l'axe du promontoire isobarique. Au sud de cet axe et de son prolongement, la pluie diminue beaucoup sur un étroit espace, pour cesser à peu près complètement dans toute une zone étendue qui correspond à ros provinces méditerranéennes (au sud de Briançon, de Gap, de Die), puis à la vallée de a Saône et du Rhône, abritée par le Massif Central jusque vers Mâcon ou Chalon (de moins en moins du sud au nord à cause de l'abaissement du relief). Voir les cartes présentées en même temps que cet exposé (fig. 3 et 4).

Lors des cas les plus fréquents, semble-t-il, et les plus typiques, le cyclone passe exactement entre l'Ecosse et la Scandinavie, le promontoire anticyclonique occupe toute l'Espagne et empiète sur l'Aquitaine; les rivières les plus atteintes sont le Rhône supérieur, l'Aar et le Rhin, la Moselle, la Meuse, la Seine, la Marne, etc. Les localisations isobariques et pluviales que comportent ces phénomènes se ressemblent d'une façon étonnante, dans des centaines d'exemples. Au point que, si l'on montre en cachant la date, les cartes d'isobares et d'isohyètes pour les averses de janvier 1899, 1910, décembre 1925, novem. bre 1944, etc., par exemple, le lecteur de ces dessins risque à tous coups de les prendre les uns pour les autres.

Pour que les rivières océaniques les plus méridionales: Vienne, Dordogne, Lot, etc:, parfois Adour et Midouze, soient plus impressionnées et les cours d'eau septentrionaux dans une moindre mesure, il faut que tout le système isobarique, sans changer d'allure générale, soit décalé vers le sud: la dépression passe sur le Bassin de Londres, la Manche, les Pays-Bas, et l'anticyclone n'occupe que le sud-ouest de la péninsule ibérique.

Et maintenant, voyons d'après $M$. ROTHÉ et la Météorologie Nationale, ce qui s'est passé le 28 décembre 1947

\section{II. - DEPRESSION RESPONSABLE DE LA CRUE DE LORRAINE}

\section{Vents et température}

Ce jour-là, à 6 heures, moment où devient grave la pluie qui durait depuis 2 ou 3 jours avec une force modérée, la carte du temps indique un cyclone profond $(970$ millibars en son centre, soit $728 \mathrm{~m} / \mathrm{m}$ ) excctement situé entre l'Ecosse et la pointe méridionale de la Norvège, position susceptible de déclencher la pluie océanique classique à localisation plutôt septentrionale. Mais il manque au tableau le bombement anticyclonique sur l'Espagne. Du moins cet anticyclone qu'on peut plutôt qualifier d'africain tout en occupant la péninsule ibérique, n'y dessine aucun promontoire accentué. Les isobares sont plutôt alignées d'ouest à est. C'est peut-être à cette absence de protubérance anguleuse qu'il faut attribuer l'extension peu classique de notre pluie : cependant à priori nous aurions plutôt attendu que cette disposition accroitrait l'averse sur la zone située au sud de la Seine, de la Champagne et de la Lorraine, alors que l'effet a été contraire. Ce qui prouve combien l'erreur est facile, même pour les «spécialistes».

Entre le 28 et le 29 au matin, la dépression a progressé assez peu et le même type de temps persiste sur l'Europe Occidentale. II consiste d'abord en une tiédeur très marquée, voire impressionnarite en certains points, et qui s'explique par un afflux puissant d'air tropical dans le secteur suá de la dépression, alors que le 27 encore, malgré l'établissement de la pluie par vent de sud-ouest, l'air était bien plus frais. $\mathrm{Ci}$ après les températures maxima et minima observées à certaines stations du 25 au 30 . Voir Tableau 1 en annexe.

Les températures maxima de 10 à $13^{\circ}$ le 28 , ou le 29 très tôt, avant l'arrivée du front froid, dans toute la région située à l'ouest ou au nordouest des Vosges sont significatives. Ce sont d'ailleurs les températures qu'on observe classiquement lors de ces phénomènes. Plus curieux, mais pas non plus anormal est le phénomène signalé par $M$. ROTHÉ pour la plaine d'Alsace, à 


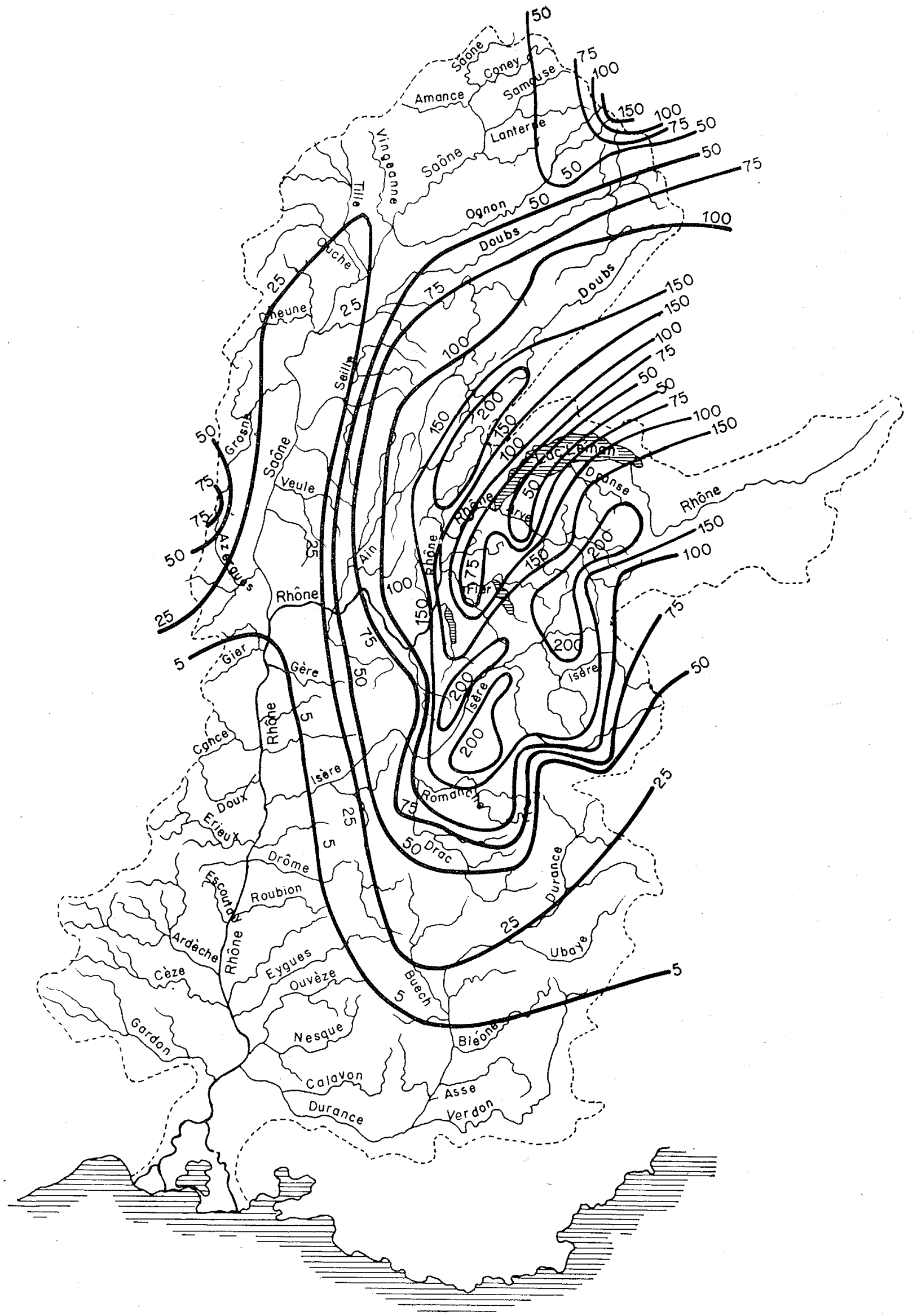

Fig. 3. - Carte de l'averse océanique du 12 au 16 janvier 1899. 


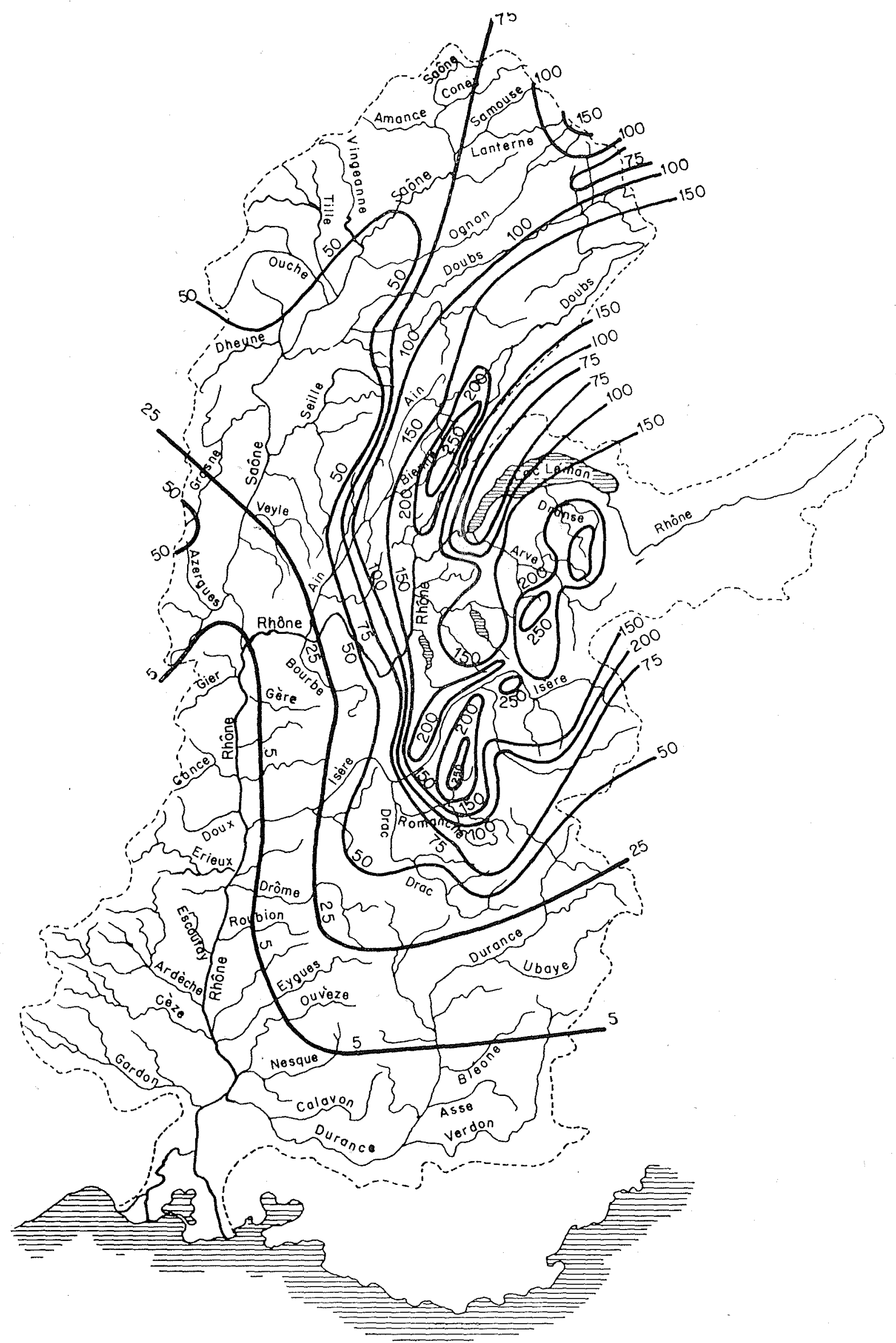

Fig. 4. - Carte de l'averse océanique du 18 au 21 janvier 1910. 


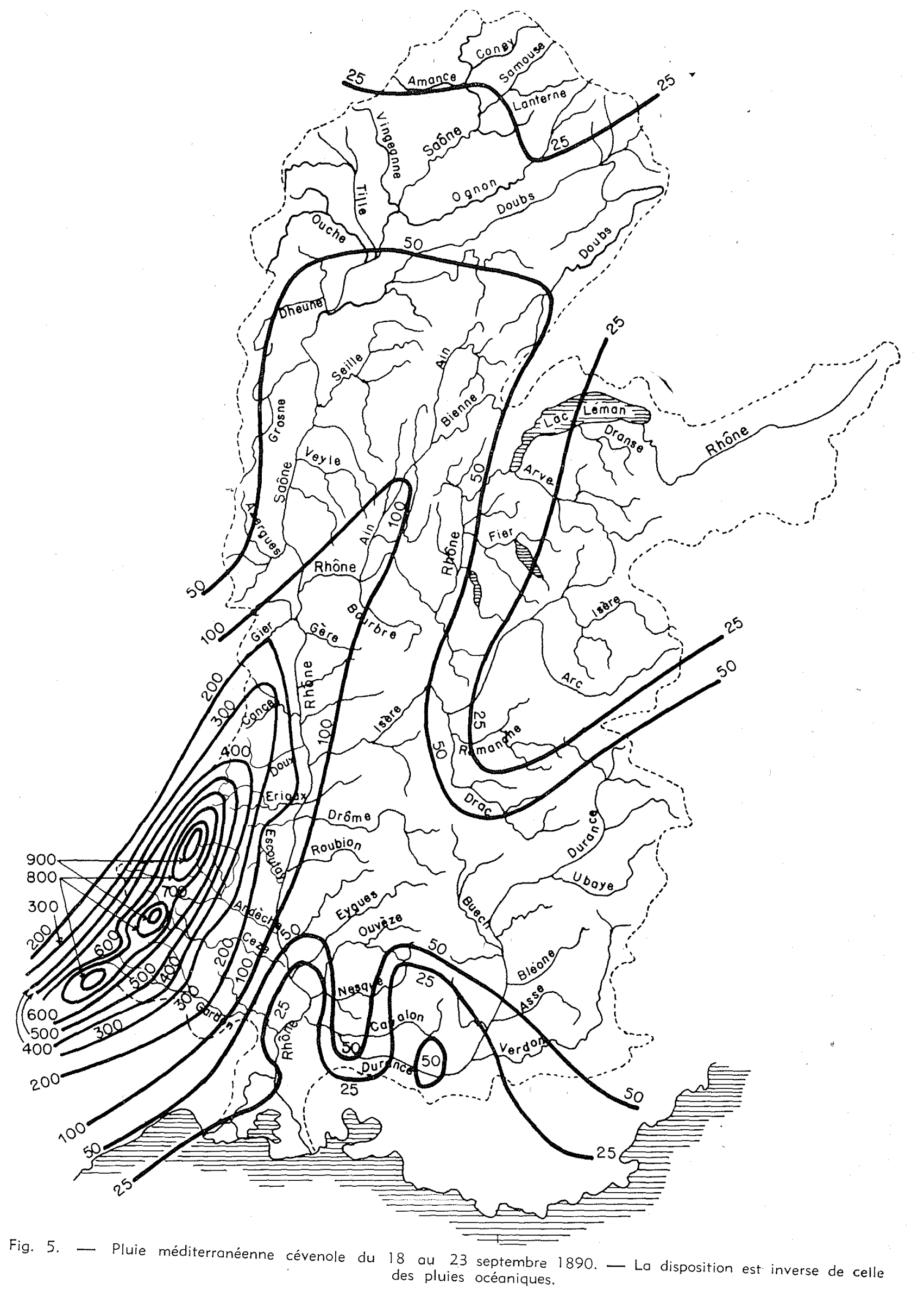


savoir le réchauffement supplémentaire produit en vertu d'un mécanisme analogue à celui du fœhn, par la descente de l'air sur le versant oriental des Vosges pendant quelques heures, le 28 : d'où un maximum de $14^{\circ} 9$ à Strasbourg et une moyenne journalière de $13^{\circ} 9$ à Colmar le même jour (maximum de $14^{\circ} 6$ seulement, mais point de rafraîchissement temporaire avec pluies comme à Strasbourg).

Durant toute la journée la plus critique, à savoir le dimanche 28 et les premières heures du 29, une très imposante tempête d'ouest ou d'ouest-sud-ouest a régné sur la plus grande partie de la France.
Le 29 au matin (vers 9 heures à Chaumont où je me trouvais), survient brusquement le front froid dont nous avons parlé plus haut et qui en général amène la fin de la pluie tiède continue en hiver; la température tomba de plusieurs degrés; même à midi à Strasbourg, elle est à 12" au-dessous du chiffre de la veille. II neige par place et même il gèle, et ce refroidissement s'occentue encore le 30 . Le ruissellement est conjuré

Nous devons insister sur la chance qu'on a eue dans cet arrêt brusque de la pluie et de la fonte, après un paroxysme guère plus long que 24 heures. On doit juger comme très possible

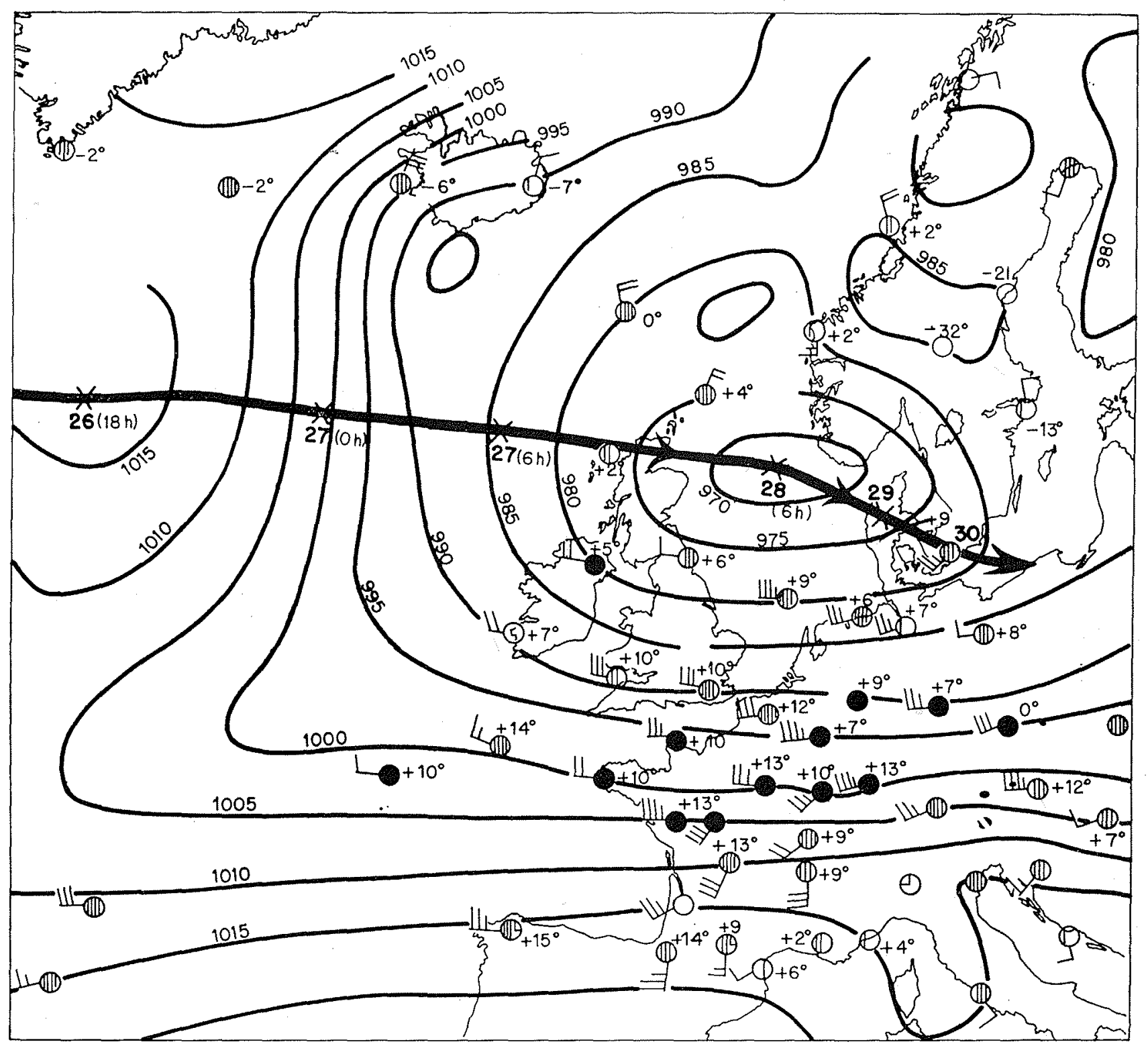

Fig. 6. - Situation barométrique le 28 décembre 1947 à 6 heures (d'après le bulletin quotidien de renseignements de I'E.C.M.). Les points noirs indiquent la position du corps pluvieux; les chiffres indiquent les températures en chaque station: les positions successives du centre cyclonique sont indiquées sur son axe de propagation du 26 au 30 décembre 1947. 


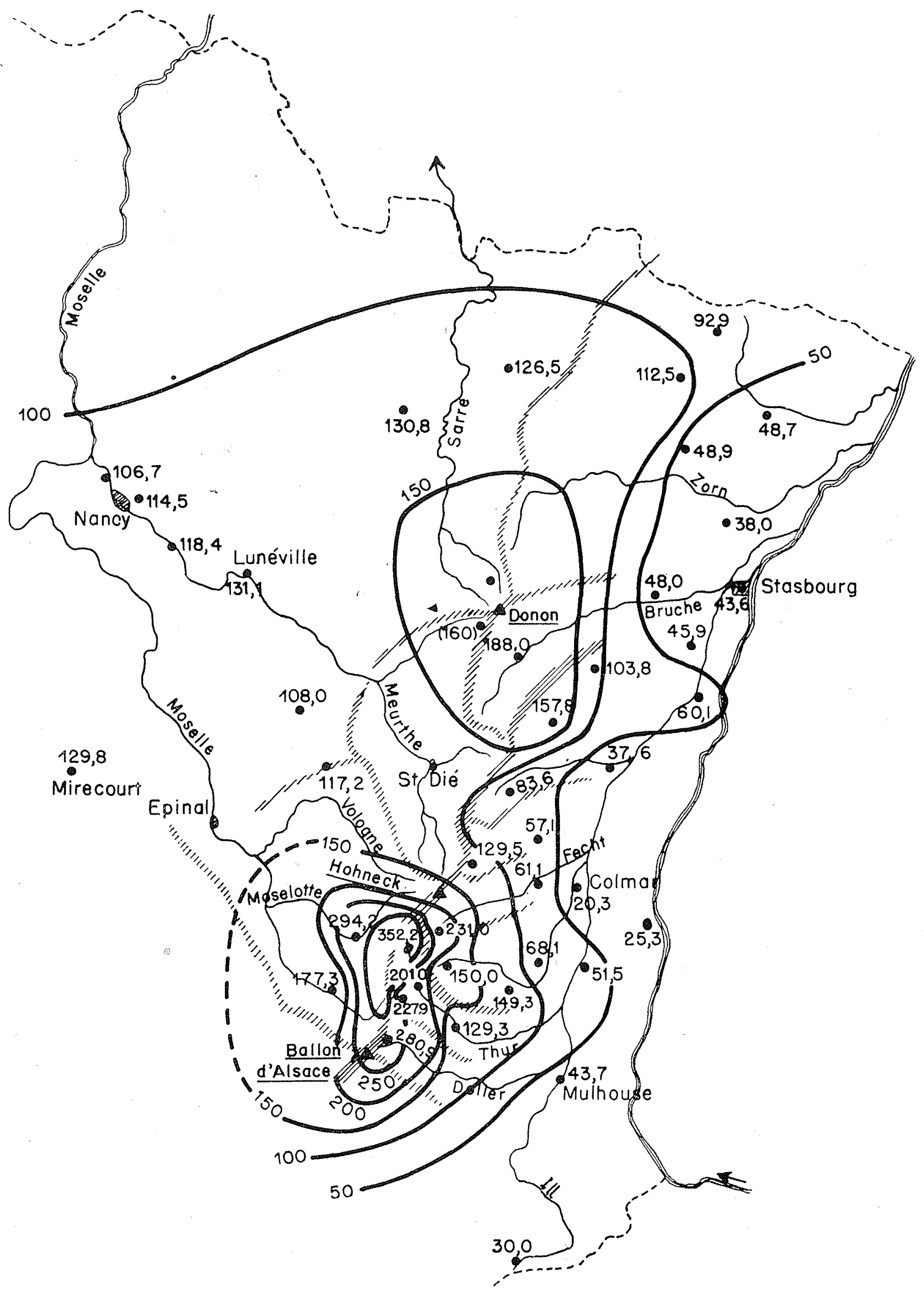

Fig. 7. - Pluie recueillie du 26 décembre 1947 (7 heures) au 30 décembre 1947 (7 heures) 


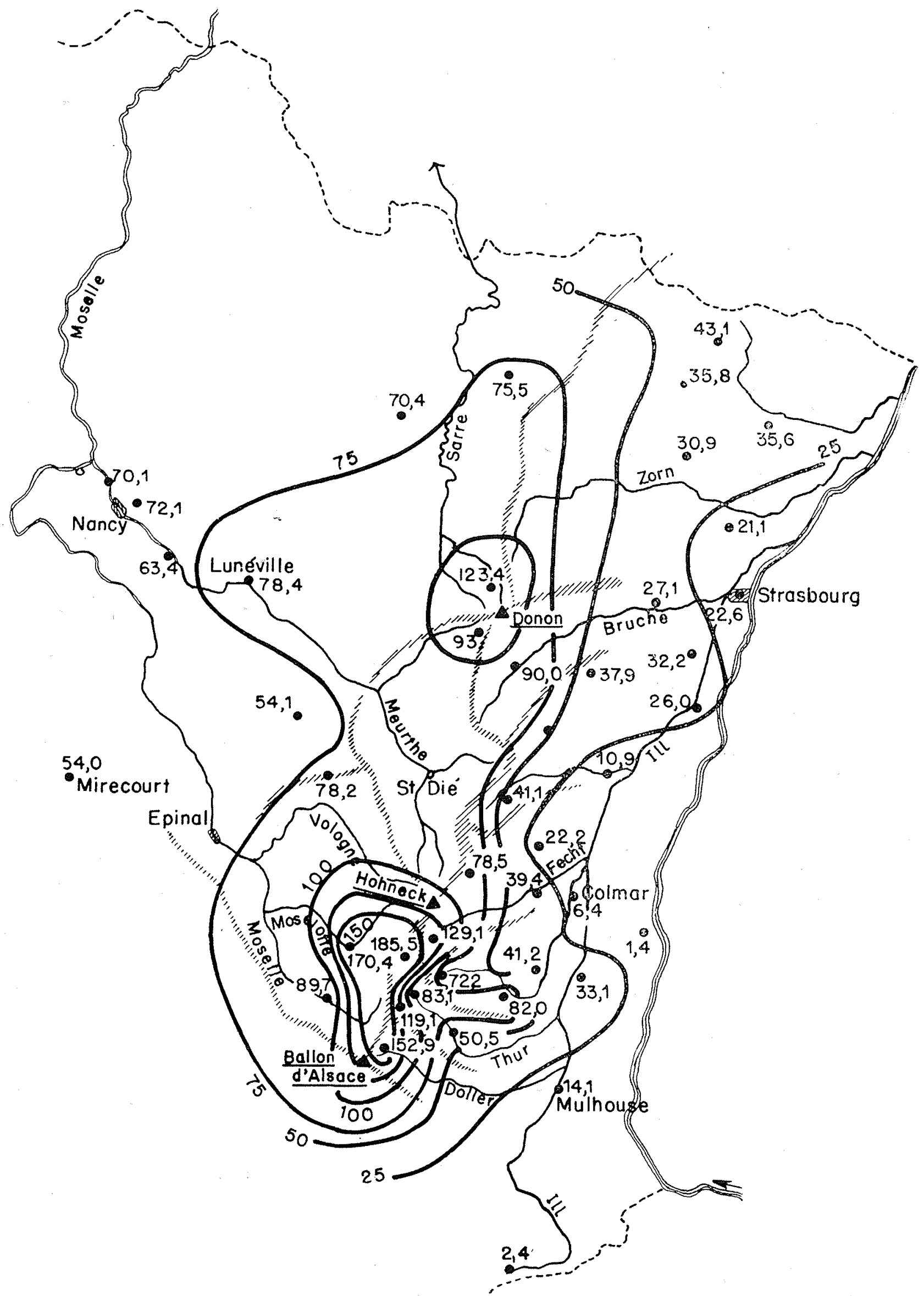

Fig. 8. - Pluie recueillie du 28 décembre 1947 (7 heures: ou 29 décembre 1947 (7 heures) 
une évolution atmosphérique plus lente qui eût prolongé la grande averse de 12 heures encore. Dans ce cas, la catastrophe eût été terrifiante: par exemple, le maximum eût été accru de 0 m. 75 d̀ Nancy, de 1 m. 50 à Metz.

\section{III. - LA PLUIE}

\section{a) - Les relevés :}

D'après les données fournies par M. SAMSON, on peut caractériser les chutes de pluie par les valeurs suivantes : (Fig. 7 et Tableau II en an. nexe).

Nous devons signaler que nous avons ajouté aux pluies du jour le plus arrosé $(7$ heures le 28 à la même heure le 29) les chutes très faibles du jour suivant, tout en sachant qu'une partie de celles-ci tombées en neige n'ont pas contribué au ruissellement. Mais quelques millimètres qui ont atteint le sol entre 7 et 9 heures le 29 peuvent être associés à l'averse principale. Enfin, pour certains postes, nous avons admis d'après les apparences que les observations ont été faites plus tard que 7 heures, à midi par exemple, et que de ce fait on a attribué à la pluie maxima de 24 heures moins d'eau qu'elle en a comporté, et à celle des 24 heures précédentes, un total trop grand. Ce doit être le cas pour Mirecourt où, au lieu de 58 , puis $54 \mathrm{~m} / \mathrm{m}$, il a dû tomber environ 30 à 35 millimètres du 27 au 28 et 77 à 82 du 28 au 29.

En outre, il est certain (voir plus loin) que dans la région de la Meuse moyenne, de l'Aire, et de Bar-le-Duc (Ornain, Saulx), c'est-à-dire Marne), il y eut effectivement de très grosses chutes dès le 27.

Enfin, nous ajoutons au tableau de ces pluies quelques indications sur celles qui sont tombées lors de deux autres crues lorraines remarqua. bles, en janvier 1910, date d'une des inondations océaniques les plus célèbres arrivées dans l'ensemble de la France, et en décembre 1919, date du maximum le plus haut connu de la Moselle à Epinal avant l'inondation récente. (En outre, on observa des crues graves du Doubs, de la Saône, du Rhône, de la Seine).

\section{b) - Moyenne sur l'ensemble des bassins :}

Des calculs approximatifs montrent que, du 25 au 29, il est tombé à peu près $125 \mathrm{~m} / \mathrm{m}$ sur la Meurthe, 145 ou 150 sur la Moselle avant la Meurthe, 120 sur la Meuse supérieure jusque vers Dun ou Stenay, 170 à 180 sur la Moselle avant Epinal, - plus le 120 sur la Marne jusque vers St-Dizier, 110 à 120 sur la Sarre, etc. Ces chiffres, quoique fort imposants et très supé- rieurs en général, sauf peut-être pour la Moselle avant Epinal, à ceux de 1910 et de 1919, n'égalent pas tout à fait les valeurs de ceux qu'on a trouvés lors de certaines crues océaniques dans les Préalpes du Nord et le Jura, par exemple, en quelques jours :

\begin{tabular}{|c|c|c|}
\hline & Ain & $\begin{array}{l}\text { Rhône y compris } \\
\text { I'Ain entre } \\
\text { Genève et Lyon }\end{array}$ \\
\hline $5-10$ mars 1896 & 238,3 & 100,3 \\
\hline $18-21$ janvier 1910 . & 150 & 129,9 \\
\hline $22-25$ décembre 1918 & 129,6 & 104,9 \\
\hline 13 - 15 février 1928. & 169,7 & 124,5 \\
\hline
\end{tabular}

NOTA. - Pour tenir compte de l'influence du relief, il faudrait majorer ces moyennes, peutêtre de un septième pour l'Ain, de un huitième pour l'ensemble du bassin entre Genève et Lyon.

\section{c) - Pluies maxima d'un jour sur les Hautes- Vosges (fig. 8, carte prêtée par M. ROTHÉ)}

Mais l'averse en question se présente comme bien plus remarquable si l'on considère les chutes maxima et les moyennes d'un jour (du 28 à 7 heures au 29 à 7 heures comme nous l'avons déjò précisé).

Examinons d'abord les relevés effectués dans les Vosges, zone la plus arrosée à cause de l'ascension des nuages. On trouve:
89,7 à Ramonchamp
170,4 à Saulxures
185,5 à Wildenstein
152,9 au Lac d'Alfeld
129 à Mittlach.

Ces chiffres paraissent un peu inférieurs dans l'ensemble aux maxima journaliers de 1919. Et le record vosgien appartient à la pluie mémorable de janvier 1910 avec $192,4 \mathrm{~mm}$. en 1 jour au lac de la Lauch.

Valeurs très imposantes pour des pluies océcniques. Presque toujours les grosses averses jurassiennes et préalpines fournissent des maxima moindres. Par exemple : 100 à 125 ou très rarement 150 millimètres. Cette différence doit tenir au fait que le Jura et les Préalpes sont protégés quelque peu contre les systèmes nuageux occidentaux par les écrans du Massif Central.

Retenons que les précipitations tombées sur les Hautes-Vosges les 28-29 décembre 1947, ne suffisent point, malgré leur puissance, d̀ expliquer la gravité exceptionnelle de la crue, si on les compare à celles de 1919 et de 1910 .

Cependant on notera que dans les Vosges septentrionales vers le Donon, les pluies, quoique très inférieures à celles qu'on observa plus au sud, à cause d'une saillie moindre du relief, ont dépassé, semble-t-il, les records précédents pour cette zone. Et ce fait a dû contribuer sensible- 
ment à la gravité de la crue sur la Sarre et sur la Meurthe qui émanent directement ou par des affluents (la Plaine pour la Meurthe), de ce massif.

\section{d) - Pluie maxima d'un jour dans l'avant-pays lorrain}

Et nous arrivons au trait le plus distinctif de cette pluie à savoir son intensité tout à fait anormale sur le plateau lorrain et l'Est du bassin de Paris : à savoir, 70 à 80 millimètres, peut-être plus par places, sur une très vaste étendue généralement bien moins arrosée (voir les chiffres de 1910 et de 1919) lors des phénomènes classiques même formidables de ce genre. Ces abats d'eau sont exceptionnels en hiver pour des zones sans relief bien saillant. Ils se sont étendus jusqu'au delà de Nancy, de Chaumont, de Neufchâteau (3), de Commercy, de Ligny-en-Barrois, de Montfaucon, etc. Ils onf' même frappé à l'Ouest et au Nord-Ouest de Nancy une zone qui forme, de par le tracé des isohyètes, un pro. montoire étendu jusqu'à l'Ouest de la Meuse vers Verdun.

On ne saurait trop insister sur le rôle capital que joua dans le phénomène cette précipitation puissante concentrée sur un jour et qui, durant ce temps, fournit en moyenne plus de 800 à 900 lit. sec. par $\mathrm{km}^{2}$ sur plus de $10.000 \mathrm{~km}$. C'est elle qui rendit tout à fait désastreux les maxima déjà très redoutables formés à l'issue des Vosges. Et rappelons à ce propos que la crue catastrophique de mars 1930, un des plus funestes événements hydrologiques survenus en France, a dû sa virulence bien plus à 100 ou 120 millimètres tombés en 24 heures sur l'avant-pays du Tarn, de l'Aveyron, de l'Agout, au delà de la Montagne Noire et des Causses, qu'aux précipitations plus fortes, mais cependant moins anor. males relevées sur les Massifs qui séparent le réseau de la Garonne des bassins méditerranéens.

\section{e) - Maxima pluvieux remarquables de 2 jours de chaque côté de la Meuse moyenne.}

Un autre fait très original consiste dans l'abondance des chutes d'eau tombées officiellement en 48 heures (peut-être en réalité en un temps un peu moindre) dons une zone située vers Commercy, Bar-le-Duc, Damvillers; Montfaucon, etc. de chaque côté de la Meuse. Ces pluies ont atteint jusqu'à $144 \mathrm{~mm}$. en 2 jours à Montfaucon, 133

(3) Comme nous l'avons dit plus haut et d'après certaines crues locales, nous soupçonnons que les maxima d'une journée à Neufchâteau et à Mirecourt ont dépassé sensiblement les 54 ou $58 \mathrm{~mm}$. annoncés par les observateurs locaux. à Ligny-en-Barrois. Nous ne nous étonnerions pas qu'en ces lieux elles constituent des records difficilement égalables. La région de la Sarre moyenne a été aussi très arrosée pendant plus d'un jour, mais avec une intensité moindre.

Quant d̀ la cause de cette abondance extraordinaire, mémorable, dans l'avant-pays peu élevé, elle consiste certainement dans une ascension continue de l'air chaud pendant les 1 ou 2 jours considérés sur les zones en question. Faute d'un relief qui qurait justifié cette montée, on doit l'attribuer à des phénomènes frontaux. Notre correspondant $M$. ROTHE croit qu'il faut ici invoquer l'action du front froid qui a surgi brutalement, comme nous l'avons vu, à l'arrière de la masse d'air chaud et qui aurait forcé cette dernière à s'élever par poussée en biseau. Cette explication peut être plausible. Nous avouons qu'elle ne nous convainc point entièrement. Les pluies de front froid durent moins que les précipitations ici examinées. Ou lorsqu'elles persistent, elles s'accompagnent à la surface du sol d'un rafraîchissement marqué qui transforme fort souvent les précipitations en neige pendant I'hiver. Dans le cas considéré, il semble que l'air ait été très doux durant toute la grosse pluie. Nous inclinons à expliquer le phénomène par une convergence relative qui, sur la région basse sinistrée, aurait contraint les vapeurs chaudes à monter. Mais ce n'est qu'une supposition peut. être peu scientifique. Et il faudrait encore détailler, avec preuves à l'appui, comment la chose se serait faite.

\section{f) - Médiocrité des chutes d'eau au sud de la Lorraine et de I'Alsace. (4)}

Nous rattachons cette anomalie à la consta. tation frappante qu'au sud de la Lorraine et des Vosges, les précipitations sont devenues modérées ou médiocres à partir d'une faible distance au delà de la région très arrosée. De la sorte, la Saône supérieure, sauf dans sa partie vosgienne, et le Doubs n'ont pas reçu d'averses susceptibles de les gonfler puissamment. Ceci mérite considération tout autant que l'intensité de l'averse sur l'avant-pays lorrain. Tout s'est passé comme si ce dernier phénomène avait eu pour compensation le premier. Insistons sur le fait que $d^{\prime}$ habitude lors de ces crues (et notamment en janvier 1910 qui vit une inondation désastreuse du Doubs), le Jura et les Préalpes du Nord sont très violemment arrosés en même temps que les Vosges. En somme, par cette concentration frontale de l'averse sur un secteur limité, comme par une certaine indépendance à l'égard du relief, notre pluie océanique, si classique par la dépres-

(4) Bien entendu, la plaine d'Alsace elle-même protégée par l'écran vosgien fut peu arrosée. 
sion responsable, par le vent et par les températures, présente des caractères très originaux.

\section{g) Les pluies préparatoires}

Enfin le lecteur attentif à notre tableau numérique aura certainement remarqué l'abondance relative des chutes d'eau préparatoires qui tombèrent du 25 au 27 . Elles atteignirent 30 à $50 \mathrm{~mm}$. au total sur l'avant-pays de la Moselle et de la Meurthe et là elles frappèrent un sol déjà imbibé par des fontes précédentes de neige. Lorsque survint l'averse décisive qui commença le 28 au matin, la saturation de la terre empêcha ou restreignit beaucoup l'infiltration; et tout donne à croire que sur maintes surfaces réceptrices, le coefficient d'écoulement atteignit 80 ou $90 \%$ sinon plus pour la pluie des dernières 24 heures. Cette préparation très effective par la saturation est une des causes capitales du désastre.

\section{IV. - LES FONTES DE NEIGE}

Et nous en arrivons au phénomène qui, selon une opinion populaire presque immanquable lors des crues d'hiver, aurait été le principal responsable de l'inondation étudiée dans ce mémoire, à savoir: la fonte des neiges.

Avant de présenter des chiffres précis sur l'épaisseur de la lame d'eau qui aurait été libérée lors de la pluie par la fonte des neiges, nous noterons tout de suite que cette quantité aurait dû correspondre, pour égaler l'apport pluvial, à une tranche neigeuse jamais observée sans doute dans une plaine française. En effet, nous avons vu que la pluie totale a fourni quelque $125 \mathrm{~mm}$. avant Nancy, 135 ou 140 sur la Meurthe et la Moselle en amont de leur confluent. Une tranche de neige qui aurait contenu une lame d'eau de fusion comparable à ces totciux aurait dû représenter selon sa densité, 1 mètre à 1 mètre 40 sur toute l'étendue de ces bassins. Or, n'importe quel habitant doué d'observation visuelle et de raisonnement, savait qu'au début de la pluie préparatoire il n'y avait plus de neige dans l'Est de la France en dehors des montagnes Vosgiennes. Des observations nombreuses recueillies par $M$. RO. THE, montrent, pour plus de précision, que la neige avait disparu le 27 au-dessous de 600 mètres d'altitude. Et ceci suffit à prouver que le rôle de la fonte n'a pu être que secondaire. Car d'après des planimétrages du même auteur, les surfaces plus hautes que 600 mètres n'occupent dans le bassin de la Moselle et de la Meurthe réunies que $960 \mathrm{~km}^{2}$, contre 6800 avant Frouard. Or, sur ces $960 \mathrm{~km}^{2}$, la fonte a été effectivement très intense, le 28 et au début du 29. M. ROTHE, qui faisait du ski sous cette tourmente a contemplé le phénomène, et il ne cherche point à le minimiser. Mais enfin, durant les 30 heures les plus critiques, d'après les chiffres journaliers fournis par ce savant, (tableau III), la fonte n'a nulle part dépassé 50 centimètres (valeurs trouvées à la Schlucht [1150 m.], au lac Blanc $[1050 \mathrm{~m}$.$] , au Champ de Feu$ $[1000 \mathrm{~m}$.$] ), tandis que la fusion représenta$ 40 centimètres au Grand Ballon ( $350 \mathrm{~m}$.) puis à Schaefertal $(1200 \mathrm{~m}$.), 30 centimètres à Welschbruch $(776 \mathrm{~m}$.$) , et moins ailleurs. On peut$ donc admettre que sur $960 \mathrm{~km}^{2}$, i! a fondu de 20 d̀ 50 centimètres, soit $35 \mathrm{~cm}$. en moyenne. En admettant une densité nivale considérable pour l'époque, cela représente au plus $50 \mathrm{~mm}$. d'eau de fusion. Donc, avant Frouard, la neige aurait fourni au plus, du 25 au 29, 48 millions de $\mathrm{m}^{3}$ contre plus de 900 millions résultant de la pluie. Et si l'on considère seulement la pluie décisive d'un peu plus d'un jour pendont lequel s'est concentrée presque toute la fonte, on trouve, contre 48 millions de $\mathrm{m}^{3}$ dus à la neige, au moins 575 et plutôt 600 à 625 millions de $\mathrm{m}^{3}$ donnés par la pluie, soit encore 12. à 13 fois plus, et 10 fois plus dons le cas sans doute invraisem. blable où la densité de la neige aurait comporté un équivalent de 60 millimètres d'eau de fusion.

La cause est donc entendue, bien que nous ne nous flattions pas que cette démonstration convainque certains fanatiques obstinés du rôle primordial de la fonte.

$D^{\prime}$ ailleurs, plus on se rapproche des Vosges et plus s'accroît le rôle de l'appoint nival dans le phénomène. Car la surface enneigée au début fut la même pour des bassins totaux bien plus petits. Et le renforcement considérable des pluies sur les Vosges n'a point compensé l'augmentation en pourcentage de la partie soumise à la fonte.

Par exemple, pour la Moselle en amont d'Epinal, il se peut que la neige ait donné $50 \mathrm{~mm} \times$ $600 \mathrm{~km}^{2}$, soit 30 sinon 35 millions de $\mathrm{m}^{3}$. Mais là encore la pluie du jour le plus critique a fourni au moins $100 \mathrm{~mm} . \times 1015 \mathrm{~km}^{2}$ et probablement plus : soit 100 à 120 millions de $\mathrm{m}^{3}$. Donc, même en cet endroit, l'apport nival n'aurait guère dépassé, ou maximum; le tiers du contingent pluvial, ni le quart de l'eau atmosphérique mise en jeu par le ruissellement. Et pour la Meurthe qui vient de hauteurs vosgiennes moins saillantes que pour la Moselle et qui ne doivent guère occuper au-dessus de 600 mètres plus de $300 \mathrm{ou}$ $400 \mathrm{~km}^{2}$, l'influence absolue et relative de la neige a été plus faible que pour l'autre rivière.

Ceci dit, il s'impose à l'attention qu'un supplément liquide de 30 à $35 \%$ jeté dans le lit de la Moselle à Epinal a rendu catastrophique une crue qui, sans ce facteur, aurait fait des dégâts 
supportables. A Nancy, la neige a pu ajouter 6 à $10 \%$ d'eau à la pointe maxima, et plus pour la Moselle à Toul, moins évidemment pour la rivière à Metz. L'absence de ce renfort aurait rendu dans cette région si éprouvée la crue moins épouvantable, sans supprimer, à beaucoup près, sa malfaisance. En ce qui concerne la Sarre, la neige n'a pour ainsi dire pas compté $180 \mathrm{~km}^{2}$ au-dessus de 600 mètres). Et pour la Meuse, son rôle a été rigoureusement nul. Ces raisonnements ne doivent guère comporter le risque d'inexactitudes majeures. Ils permettent d'apprécier assez bien dans quelle mesure la crue lorraine a été cousée par la fonte des neiges.

\section{V. - CONCLUSIONS GENERALES SUR LES CAUSES}

En définitive, la grande crue de décembre 1947 sur la Moselle et la Meurthe, rivières les plus touchées par le phénomène, a- eu pour cause les facteurs suivants :

$1^{\circ}$ - Lors d'une situation atmosphérique conforme dans ses grandes lignes à celles qui causent toutes les averses océaniques classiques, des pluies formidables, quoique déjà égalées, ont frappé les Vosges.

$2^{\circ}$ - Des fontes de neige elles-mêmes anormales ont accru la masse $d^{\prime}$ eau écoulée d'un tiers peut-être à Epinal, de 8 à $10 \%$ au plus à Nancy, de 10 à $12 \%$ peut-être à Toul, selon des évaluations assez larges.

$3^{\circ}$ - Des pluies préparatoires de 2 à 3 jours avaient saturé complètement le sol. Et cette influence a aggravé beaucoup le ruissellement, donc la crue.

$4^{\circ}$ - Le facteur le plus anormal et le plus désastreux a été la chute de 70 à 80 millimètres en un jour sur un large avant-pays, toujours bien moins arrosé lors des phénomènes de ce genre. D'où crues terribles de la Vezouse, du Madon, de la Mortagne, de la Plaine, et renforcement de plus en plus redoutable pour les maxima des rivières principales, en aval des Vosges.

Sans cette caractéristique pluviale, il n'y aurait point eu de catastrophe en dehors de la région vosgienne ou de ses abords immédiats.

\section{VI. - LES CRUES}

De fait, d'après des renseignements provisoires aimablement communiqués par $M$. SIEGFRIED, Ingénieur en Chef par intérim des Ponts et Chaussées à Nancy, et d'après les journaux, les records précédents de hauteurs n'ont pas ou guère été dépassés sur la Meurthe avant Lunéville; et à Epinal, il se peut, d'après des renseignements non définitifs, que la cote de 1947 n'ait pas excédé de plus de 10 centimètres celle de 1919 (0 m. 60 d'après d'autres informations). Mais à Toul, la Moselle cota $4 \mathrm{~m} .60$ contre 3,75 en 1919; à Millery $6 \mathrm{~m} .40$ contre $5 \mathrm{~m} .25$; à Metz $8 \mathrm{~m}$. 90 contre $7 \mathrm{~m}$. 20. Pour lo Meurthe à Malzéville (Nancy) l'excédent sur 1919 n'a été que de 40 centimètres $(4 \mathrm{~m}, 80$ contre $4 \mathrm{~m} .40$, et $4 \mathrm{~m} .60$ en 1778). Mais I'inondation bien plus forte, sans précédent à beaucoup près, survenue en 1947 nous donne à supposer que cette différence est trop faible pour permettre une comparaison sûre entre les diverses crues.

Lors de celle de décembre dernier, une partie notable du débit a emprunté le canal d'où elle s'est déversée dans la ville. Sans cet accident, elle aurait passé par le lit ordinaire et sans doute aurait porté à $5 \mathrm{~m}$. 20 sinon plus, au lieu de $4 \mathrm{~m}$. 80 la hauteur maxima observée à l'échelle de Malzéville.

Nous espérons que le rapport attendu des spécialistes nancéiens nous fournira sur ces différences et les perturbations de hauteurs toutes les précisions désirables, et de même des évaluations fondées, sur les débits maxima.

$D^{\prime}$ après les calculs de $M$. DUMAS, Ingénieur au Laboratoire d'Hydraulique de I'Institut Polytechnique de Grenoble, nous croyons savoir que le maximum à Epinal aurait voisiné $1100 \mathrm{~m}^{3} / \mathrm{sec}$. chiffre qui représente à peu près 1000 lit. sec. par $\mathrm{km}^{2}$, et ne nous étonne pas du tout d'après l'intensité de l'apport pluvial et nival. (Pour la surface réceptrice en amont $d$ 'Epinal, nous n'avons que des chiffres discordants: 1015 à $1250 \mathrm{~km}^{2}$ )

D'après les causes, il ne nous surprendrait point que le maximum ait représenté 400 à 450 lit. sec. par km² soit 1200 à $1350 \mathrm{~m}^{3} / \mathrm{sec}$. à Nancy; et 450 à 500 lit. sec. par $\mathrm{km}^{2}$ sur la Moselle vers Liverdun, soit 1700 à $1900 \mathrm{~m}^{3} / \mathrm{sec}$. En aval, jusque vers Metz le flot le plus puissant aurait roulé quelque 2500 et peut-être $3000 \mathrm{~m}^{3} / \mathrm{sec}$. Mais ce sont des estimations tout à fait grossières sans bases hydrométriques sérieuses, et que nous présentons faute de mieux.

Nous croyons encore savoir que la Meuse, vers Commercy, Verdun et un peu en aval, a battu tous les records. A Verdun on cota $4 \mathrm{~m}$. 30 et la rivière débita $700 \mathrm{~m}^{3} / \mathrm{sec}$. d'après les Services Officiels. Mais nous ne connaissons guère les chiffres antérieurs.

La crue fut aussi anormale à Bar-le-Duc sur l'Ornain, encore plus forte sur la Sarre et sur les rivières des Vosges orientales aboutissant à I'lll. Mais sur la Meuse et la Moselle inférieures, il nous semble qu'elle n'a point égalé à bequcoup près, surtout pour la Moselle, les records de janvier 1926, car sur la partie septentrionale de ces bassins, la pluie paraît avoir été aussi modérée que sur la Saône ou le Doubs. 
TABLEAU

Températures minima et maxima observées du 25 au 30 décembre 1947

\begin{tabular}{|c|c|c|c|c|c|c|c|}
\hline \multicolumn{2}{|c|}{ Station } & 25 & 26 & 27 & 28 & 29 & 30 \\
\hline \multirow{2}{*}{\multicolumn{2}{|c|}{ MIRECOURT }} & 5.0 & 2.2 & 3.6 & 4.0 & 4.6 & -0.4 \\
\hline & & 6.8 & 6.4 & 9.6 & 10.2 & 12 & 3 \\
\hline \multirow{2}{*}{\multicolumn{2}{|c|}{ NANCY }} & 3.9 & 3.5 & 5.2 & 10.2 & 3.0 & 1.1 \\
\hline & & 5.4 & 7.2 & 10 & 12.8 & 12.8 & 3.2 \\
\hline \multirow{2}{*}{\multicolumn{2}{|c|}{ METZ }} & 3.2 & 2.2 & 1.8 & 6.2 & 1.8 & 0.0 \\
\hline & & 6.2 & 6.4 & 10.2 & 12.3 & 8.6 & 2.8 \\
\hline \multirow{2}{*}{\multicolumn{2}{|c|}{ BELFORT }} & 2.0 & 2.2 & 2.6 & 3.2 & 9.8 & -1.6 \\
\hline & & 4.0 & 7.6 & 8.0 & 13.0 & 12.8 & 0.8 \\
\hline \multirow{2}{*}{\multicolumn{2}{|c|}{ STRASBOURG }} & 3.8 & 4.2 & 2.2 & 4.6 & 5. & 0.4 \\
\hline & & 5.7 & 9.2 & 9.8 & 14.9 & 15. & 5.0 \\
\hline
\end{tabular}

TABLEAU

Hauteurs de pluie, en millimètres, observées du 25 au 28 décembre 1947

\begin{tabular}{|c|c|c|c|}
\hline S'ATTON & $\begin{array}{c}\text { Pluie prepara. } \\
\text { toire du } 25 \\
\text { alu } 27\end{array}$ & 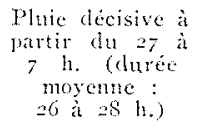 & Totat. \\
\hline
\end{tabular}

\section{Bassin de la Meurthe}

$\begin{array}{lllll}\text { Brouvelieures } \ldots & 37,3 & 87,2 & 124,5 \\ \text { Rambervillers } \ldots & \ldots & 42,7 & 65,3 & 108 \\ \text { Lunéville } \ldots \ldots & 44 & 87,1 & 131,2 \\ \text { St-Nicolas-du-Port } & 47,7 & 77,8 & 125,5 \\ \text { Glocimont . . . . . } & \text { (?) } & 93,8 & 160\end{array}$

\section{Bassin de la Moselle}

\begin{tabular}{|c|c|c|c|}
\hline Ramonchamp ..... & 99,4 & 105,4 & 204,8 \\
\hline Soulxures-s/-Mosel. & 146,4 & 178,2 & 333,6 \\
\hline Mirecourt $\ldots . .$. & 51 (?) & 90,2 & 141,2 \\
\hline$\ldots \ldots$ & 49,2 & 79,7 & 128,9 \\
\hline Champigneulles ... & 48,7 & 74,9 & 123,6 \\
\hline Metz & 27,8 & 56,8 & 84,6 \\
\hline $\begin{array}{l}\text { Crête des Vosges } \\
\text { (versant Alsace) }\end{array}$ & & & \\
\hline Wildenstein & 126,5 & 185,5 & 352 \\
\hline Lac d'Alfeld & 128 & 152,9 & 280,9 \\
\hline Mittlach .. & 101,9 & 129,1 & 231 \\
\hline Oderen $\ldots \ldots .$. & 116 & 85 & 201 \\
\hline
\end{tabular}

\begin{tabular}{|c|c|c|c|}
\hline STATION & $\begin{array}{l}\text { Pluice ureprara. } \\
\text { toire du } 25 \\
\text { at } 27\end{array}$ & 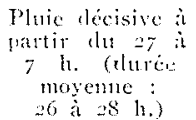 & "OTAI \\
\hline
\end{tabular}

\section{Meuse Supérieure}

Vigneulles ...... 72,3

$63,2 \quad 135,6$

Bassoncourt ..... 39,2

$43,9 \quad 83,1$

Meury 194

43,9

83,1

Bourmont ..... 33,4

69,8

103,2

Neufchâteau .... 61,5 (?) $80 \quad 141,5$

Commercy ..... $49 \quad 112 \quad 161$

Herserange $\ldots \ldots 30,8 \quad 86,5 \quad 117,3$

Damvillers ...... 50,6 117,7 (2 i.) 168,3

Montmédy ....... 9 (?) $74 \quad$ (2j.) 83 (?)

Montfaucon ...... $35 \quad$ (2 j.) $144 \quad$ (2 j.) 179

\section{Bassin de I'Aisne}

Loxéville f.... $42 \quad$ (2 i.) $129,1 \quad$ (2 j.) 171,1

\section{Bassin de la Marne}

\begin{tabular}{|c|c|c|c|c|c|}
\hline Langres . & 30,3 & & 54,2 & & 84,5 \\
\hline Chaumont & 29,3 & & 84,1 & & 113,4 \\
\hline Poissons. & 56,8 & $(2 j)$. & 93,9 & $(2 j)$ & 150,7 \\
\hline Wassy ..... & 20,2 & $(2 \mathrm{j})$. & 100,2 & $(2 \mathrm{j})$. & 120,4 \\
\hline Ligny-en-Borrois & 46 & $(2 j)$. & 133 & $(2 j)$ & 179 \\
\hline
\end{tabular}

\section{Aube}

$\begin{array}{lllllr}\text { Auberive } \ldots \ldots \ldots & 17,5 & 57 & & 74,5 \\ \text { Châtequvilloin .... } & 25,6 & & 69,3 & & 94,9 \\ \text { Montier-en-Der ... } & 19,6 & \text { (2 j.) } & 80,9 & \text { (2 j.) } & 100,5\end{array}$

\section{Saône Supérieure}

Bourbonne ..... 34,5

$87,5 \quad 122$

Prouthoy ....... 32,5

$53,2 \quad 85,7$

Scey-s/-Saône ... 23,5

46

85,7
69,5

Doubs

Pontarlier ..... 14,7

42,1

56,8

Montbéliard .... 21,7

28,1

49,8

Belfort ....... 32,8

33,6

66,4

\section{Alsace}

Mulhouse ...... 6,

Strasbourg ..... 12,3

Phalsbourg ..... 36,3

15,5

22,3

Mulhouse ....... 6,8

38,6

$69,1 \quad 105,4$

$15,5 \quad 22,3$

Sarre 25-26 déc. 27-28 déc.

( 2 jours)

Sarrebruck ...... 10,5

Courcelles-Chaussy . 14,2

104,2

114,7

Berus ...... 17,3

Tholey ....... 35,6

87,3

101,3

101,5

Marcarerie .......

114,3

118,6

149,9 
TABLEAU III

(prêté por $M$. Rothé)

Hauteur de la couche de neige (en centimètres)

du 23 au 28 décembre 1947

\section{A) - hAUTES YOSges}

\begin{tabular}{|c|c|c|c|c|c|c|c|}
\hline Station & Altitude & 23 & 24 & 25 & 26 & 27 & 28 \\
\hline Grand-Ballon .... & & 70 & 70 & 60 & 65 & 65 & 50 \\
\hline Schaefertal ..... & 1.2 & 40 & 40 & 55 & 60 & 65 & 50 \\
\hline Schlucht ....... & 150 & 40 & 40 & 45 & 60 & 80 & 60 \\
\hline Lac Blane & 1.050 & 40 & 40 & 45 & 60 & 80 & 60 \\
\hline Lac Noir. & 960 & 30 & 30 & 30 & 25 & 20 & 15 \\
\hline Lac d'Alfeld & 620 & 0 & 0 & & 0 & 20 & 0 \\
\hline Petit-Hout & & 5 & 0 & & 0 & & 0 \\
\hline Wildenstei & 580 & 0 & 0 & 0 & 0 & & 0 \\
\hline Mittlach & 540 & 0 & 0 & 0 & 0 & & 0 \\
\hline Urbès (Thann) ... & 450 & 0 & 0 & 0 & 0 & 0 & 0 \\
\hline Colmar ........ & 190 & 0 & $\because 0$ & 0 & 0 & 0 & 0 \\
\hline
\end{tabular}

(Altitude limite de l'enneigement : $600 \mathrm{~m}$. les 22 et 23 décembre; $800 \mathrm{~m}$. du 24 au 26 décembre; $600 \mathrm{~m}$. le 27 décembre!.

\section{B) - YOSGES MOYENNES}

\begin{tabular}{|c|c|c|c|c|c|c|c|}
\hline Station & Altitde & 23 & 24 & 25 & 26 & 27 & 28 \\
\hline Champ-du-Feu & 1.000 & 50 & 40 & 40 & 50 & 70 & 50 \\
\hline Welschbruch ... & 77 & 17 & 10 & 10 & 20 & 30 & 0 \\
\hline Glacimont (Donon) & 700 & 25 & 21 & 20 & 17 & 27 & 0 \\
\hline Marcorerie ..... & 450 & 0 & 0 & 0 & 0 & 0 & 0 \\
\hline Rothau ... & 334 & 0 & 0 & 0 & 0 & 0 & 0 \\
\hline Strosbourg & 140 & 0 & 0 & 0 & 0 & 0 & 0 \\
\hline
\end{tabular}

(Altitude limite de I'enneigement : $600 \mathrm{~m}$. du 23 au 27).
TABLEAU IV

Pluies antérieures

A) -17 au 20 janvier 1910 :

\begin{tabular}{|c|c|c|c|}
\hline Station & Jour & du maximum & Total \\
\hline Cornimont & $\ldots$ & 101 & 175 \\
\hline Brouvelieures. & $\ldots$ & 50 & 74 \\
\hline Epinal ...... & $\ldots$ & 37. & 91 \\
\hline Provenchère ... & $\ldots$ & 53 & 106 \\
\hline Baccarat .... & $\ldots$ & 22 & 49 \\
\hline Lunéville .... & $\ldots$ & 23 & 59 \\
\hline Nancy ...... & $\ldots$ & 27 & 52 \\
\hline Foug ......... & $\ldots$ & 24 & 52 \\
\hline Contrexéville., & $\ldots$ & 26 & 64 \\
\hline Neufchôteau .. & $\ldots$ & 34 & 74 \\
\hline Commercy ... & $\ldots$ & 31 & 65 \\
\hline Consenvoye ... & $\cdots$ & 25 & 41 \\
\hline Choumont .... & & 40 & 82 \\
\hline Langres ..... & $\cdots$ & 44 & 95 \\
\hline Bar-le-Duc ... & $\ldots$ & 30 & 61 \\
\hline Lac de la Lauch & & 192,4 & \\
\hline
\end{tabular}

B) - Décembre 1912:

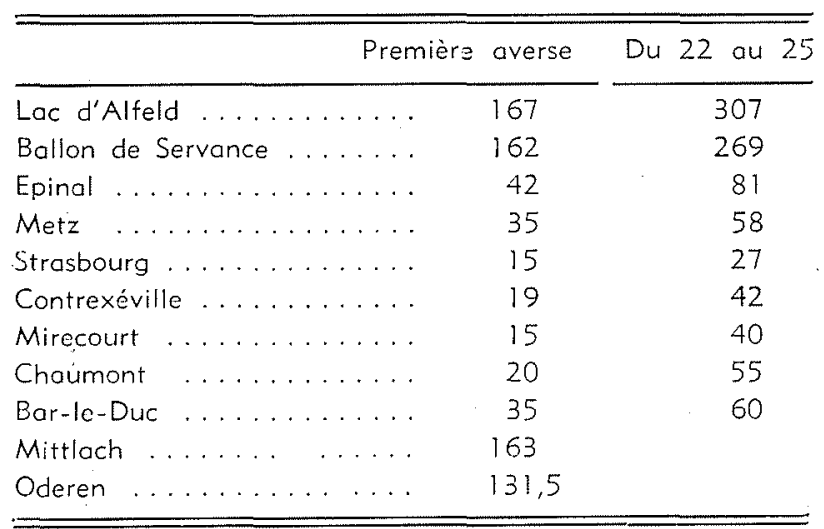

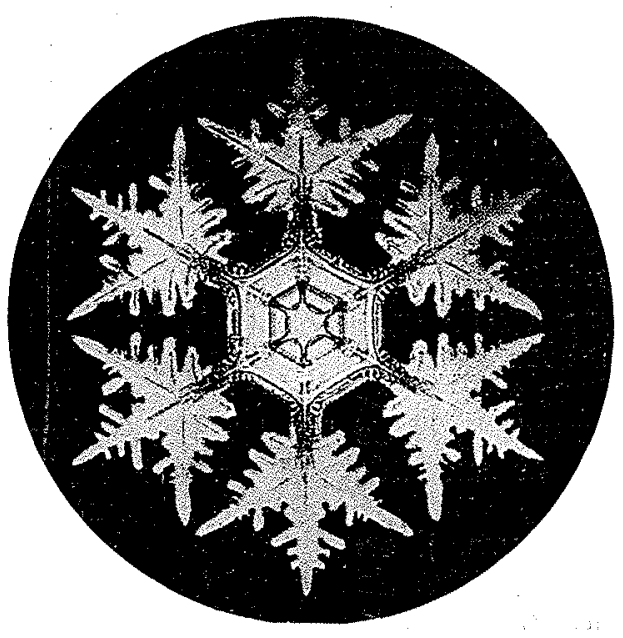

